\title{
Numero cromatico, omomorfismi e colorazioni d'un grafo.
}

\author{
Francesco Speranza $(*)(* *)$
}

A Bentamino Segre per il suo $70^{\circ}$ compleanno

Sunto. - Si studia la possibilità di considerare un dato grafo non orientato come immagine omomorfa o antiimmagine omomorja d'un opportuno grafo di numero cromatico prefissato. $S i$ definiscono poi, per un grafo colorato, gli omomorfismi semi-colorati e quelli colorati, studiando alcune proprietà delle relative categorie e costruendo un grafo colorato per il quale il gruppo degli automorfismi semi-colorati e quello degli automorfismi colorati sono isomorfi a un gruppo finito prefissato.

1. - In questo lavoro si analizzano congiuntamente proprietà di colorazione e di rappresentazione di grafi non orientati. Nel n. 2 si studia il comportamento del numero cromatico d'un grafo per effetto d'un omomorfismo, provando che, dato un grafo di numero cromatico finito, lo si può trasformare con un opportuno omomorfismo in uno di numero cromatico finito arbitrario, e si può trovare un grafo di numero cromatico arbitrario di cui quello è immagine omomorfa. Si può anzi richiedere, salvo alcuni casi, che l'omomorfismo sia iniettivo o che sia suriettivo (le dimostrazioni sono tutte costruttive).

Nel n. 3 si considera la specie di strutture di grafo colorato, definendo gli omomorfismi semi-colorati e quelli colorati, ed evidenziando alcune categorie collegate.

Si studiano poi nel n. 4 alcune relazioni fra il gruppo degli automorfismi, quello degli automorfismi semi-colorati e quello degli automorfismi colorati (che è invariante nel precedente). Si assegna una condizione necessaria e sufficiente affinchè un dato grafo non orientato si possa colorare in modo che essi siano isomorfi, e si dà una costruzione d'un grafo colorato che ammette gruppi degli automorfismi semicolorati e colorati isomorf a un gruppo assegnato, finito o numerabile.

2. - I grafi qui considerati sono grafi non orientati semplici (cioè privi di spigoli in parallelo e di lacci). In questo numero ci limiteremo a grafi di numero cromatico finito: non si chiederà invece che il grafo sia finito, salvo esplicito avviso contrario. Ordine di un grafo è la cardinalità dell'insieme dei vertici.

(*) Entrata in Redazione il 25 gennaio 1974.

(**) Lavoro eseguito nell'ambito dell'attività del G.N.S.A.G.A. del C.N.R. 
Sia $G$ un grafo non orientato e siano rispettivamente $V(G), S(G)$ (o più semplicemente $V$ e $S$ ) l'insieme dei suoi vertici e quello dei suoi spigoli: poniamo poi $\tilde{V}=\{\{u, v\}: u, v \in V\}$. Sia $\varphi(G): S \rightarrow \tilde{V}$ l'applicazione che a ogni spigolo associa l'insieme dei suoi vertici. Definiamo l'applicazione $\psi(G)$ (o più semplicemente $p$ ) di $V \cup S$ in $\tilde{V}$ tale che $\psi(x)=\varphi(G)(x)$ se $x \in S, \psi(x)=\{x\}$ se $x \in V$.

Sia poi $G^{\prime}$ un altro grafo (scriveremo anche $V^{\prime}, S^{\prime}, \tilde{V}^{\prime}, \psi^{\prime}$ in luogo di $V\left(G^{\prime}\right)$, $S\left(G^{\prime}\right), \tilde{V}\left(G^{\prime}\right), \varphi\left(G^{\prime}\right)$ rispettivamente). Un omomorfismo di $G$ in $G^{\prime}$ è un'applicazione $f: V \cup B \rightarrow V^{\prime} \cup S^{\prime}$ tale che il seguente diagramma sia commutativo:

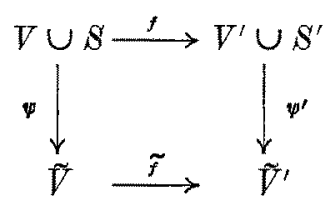

essendo $\tilde{f}$ l'estensione a $\tilde{V}$ della restrizione di $f$ a $V$.

In altri termini, un omomorfismo $f$ trasforma uno spigolo $s$ di estremi $a$ e $b$ in uno spigolo di vertici $f(a)$ e $f(b)$, o in un vertice $a^{f}=f(a)=f(b)([4],[5])$.

Un'applicazione $g: V(G) \rightarrow V\left(G^{\prime}\right)$ è la restrizione a $V(G)$ d'un omomorfismo $f$ se e solo se due vertici adiacenti in $G$ hanno per immagine due verici adiacenti o coincidenti in $G^{\prime}$. Se $G^{\prime}$ è semplice, $f$ è determinata dalla sua restrizione a $V(G)$ (si dirà che questa definisce $f$ ) e inoltre

$$
\varphi(s)=\{a, b\} \wedge f(a)=f(b) \Rightarrow f(s)=f(a)=f(b) .
$$

Vediamo brevemente il comportamento del numero cromatico d'un grafo per effetto d'un omomorfismo. Possiamo dare un grafo $G$ di numero cromatico $k$ e chiederei di costruire un grafo di numero cromatico prefissato che ne sia immagine (oppure antiimmagine) omomorfa: anzi, a questo omomorfismo possiamo chiedere inoltre di essere suriettivo o iniettivo. (Se esiste un omomorfismo suriettivo di $G$ su $G^{\prime}$, diciamo che $G$ è antiimmagine suriettiva omomorfa di $G^{\prime}$, e analogamente in casi analoghi.)

Sia $G$ un grafo di numero cromatico $k$ finito. Risolviamo i seguenti problemi.

1) Se $k>2$ trovare un grafo $G_{1}$ di numero cromatico $h<k$ tale che esiste un omomorfismo suriettivo di $G_{1}$ su $G$.

亡̀ sufficiente risolvere il problema per $h=k-1$ : l'applicazione ripetuta del metodo conduce a risolvere il problema generale.

Consideriamo una colorazione $K$ di $G$ con $k$ colori, e siano $A, B, C$ tre colori. Se $x_{i}$ sono i vertici di colore $A$ e $y_{j}$ quelli di colore $B$, per ogni spigolo $s_{i j}$ di estremi $x_{i}$ e $y_{j}$ introduciamo un nuovo vertice $z_{i j}$ e allo spigolo $s_{i j}$ sostituiamo due spigoli, $p_{i j}$ di estremi $x_{i}$ e $z_{i j}$ e $q_{i j}$ di estremi $y_{j}$ e $z_{i j}$. Il grafo $G_{1}$ così ottenuto risolve il problema. Infatti: 
a) L'applicazione $g: V\left(G_{1}\right) \rightarrow V(G)$ tale che $g\left(z_{i j}\right)=x_{i}, g(u)=u$ per ogni $u \in V(G)$ definisce un omomorfismo di $G_{1}$ su $G$ : esso è suriettivo poichè $s_{i j}$ è immagine di $q_{i j}$, mentre ogni altro spigolo o vertice di $G$ è immagine di se stesso.

b) Il numero cromatico di $G_{1}$ è $k-1$. Infatti, una colorazione di $G_{1}$ si ottiene dando a ogni vertice $x_{i}$ o $y_{j}$ il colore $B$, a tutti i vertici $z_{i j}$ il colore $C$ e a ogni altro vertice di $G_{1}$ il colore che a esso compete in $K$. Non è possibile dare, d'altra parte, a $G_{1}$ una colorazione $K^{\prime}$ con $k-2$ (o meno) colori, $L, M, \ldots, P$. Infatti, se cosi fosse, $K^{\prime}$ darebbe a ogni vertice di $G$ un colore, e si avrebbe una sua colorazione, con l'eccezione di qualche vertice $x_{i}$ e di qualche vertice $y_{j}$ i quali, essendo adiacenti, avrebbero in $K^{\prime}$ il medesimo colore. Basterebbe allora dare ai vertici $y_{j}$, che a due a due non sono adiacenti, un nuovo colore $Q$, e in tal modo si sarebbe ottenuta una colorazione di $G$ con (al più) $k-1$ colori, il che è contro l'ipotesi.

Se $k=2$ non si può, manifestamente, trovare un grafo di numero cromatico 1 che ne sia antiimmagine omomorfa suriettiva: in tal caso, la costruzione precedente conduce ad un grafo $G_{1}$ di numero cromatico non inferiore a 2 .

2) Trovare un grafo $G_{2}$, di numero cromatico $h<k$, tale che esista un omomorfismo iniettivo di $G_{2}$ in $G$.

Ovviamente il problema ha senso solo se $k \geqslant 2$ : inoltre, basta risolverlo per $h=k-1$ (cfr. il prob. 1))

Consideriamo una colorazione $K$ di $G$ con $k$ colori, e fissiamo l'attenzione su due colori $A$ e $B$, e sui vertici $\left(x_{i}\right)$ di colore $A$ e quelli $\left(y_{j}\right)$ di colore $B$. Aboliamo tutti gli spigoli aventi per estremi un vertice $x_{i}$ e un vertice $y_{j}$ : si ottiene così un grafo parziale (efr. [1]) di $G$, diciamo $G_{2}$. Esso risolve il problema.

a) L'immersione di $G_{2}$ in $G$ è un omomorfismo iniettivo.

b) $G_{2}$ ha numero cromatico $k-1$. Infatti, assegnando ai vertici $x_{i}$ e $y_{j}$ il colore $B$, e agli altri il colore che a ciascuno compete in $K$, si ha una colorazione di $G_{2}$ con $k-1$ colori. Non è d'altra parte possibile colorare con meno di $k-1$ colori $G_{2}$ : se esistesse una tale colorazione, diciamo $K^{\prime}$, consideriamo la colorazione di $G$ nella quale ai vertici $y_{j}$ si dà un nuovo colore, mentre tutti gli altri hanno il colore che loro compete in $K^{\prime}$ : in tal modo si otterrebbe una colorazione di $G$ con meno di $k$ colori, contro l'ipotesi.

3) Trovare un grafo $G_{3}$ di numero oromatico $h>k$, tale che esista un omomorfismo suriettivo ${ }^{3+t} d i G_{3}$ su $G$.

Sia $G^{*}$ un grafo connesso di numero cromatico $h$, avente un vertice a (e nessun altro elemento) comune a $G$. Allora il grafo $G_{3}$, tale che

$$
\begin{aligned}
& V\left(G_{3}\right)=\nabla(G) \cup \nabla\left(G^{*}\right), \quad S\left(G_{3}\right)=S(G) \cup S\left(G^{*}\right), \\
& \varphi\left(G_{3}\right)(s)=\varphi(G)(s) \text { per } s \in S(G), \quad \varphi\left(G_{3}\right)(s)=\varphi\left(G^{*}\right)(s) \text { per } s \in S\left(G^{*}\right),
\end{aligned}
$$

risolve il problema. 
a) L'applieazione $g: V\left(G_{3}\right) \rightarrow V(G)$ tale che $g(x)=x$ per $x \in V(G), g(x)=a$ per $x \in V\left(G^{*}\right)$ definisce un omomorfismo suriettivo di $G_{3}$ su $G$.

b) $G_{3}$ ha numero cromatico $h$. Esso infatti, contenendo un sottografo di numero cromatico $h>k$, non può avere numero cromatico $\leqslant k$. D'altra parte, fissata una colorazione $K$ di $G^{*}$, possiamo colorare $G$ con $k$ dei colori di $K$ (rispettando ovviamente il colore di $a$ ), e quindi $G_{3}$ è colorabile effettivamente con $h$ colori.

4) Trovare un grafo $G_{4}$ di numero cromatico $h<k$ e un omomorfismo suriettivo di $G$ su $G_{4}$.

Il problema ha senso solo se $k \geqslant 2$ : risolviamolo allorehè $h=k-1$ (cfr. il problema 1)).

Consideriamo una colorazione $K$ di $G$ con $k$ colori, e fissiamo l'attenzione su due colori $A$ e $B$ : sia $b$ un vertice di colore $B$. Consideriamo il seguente grafo $G_{4}$ : i suoi vertici sono i vertici di $G$, eselusi quelli che in $K$ hanno colore $A$; gli spigoli di $G$ che non hanno estremi di colore $A$ sono pure spigoli di $G_{4}$, e inoltre, se in $G$ v'è uno spigolo di estremi $x_{i}$ e $y$, dove $x_{i}$ ha colore $A$ e $y$ ha colore diverso da $A$ e da $B$, in $G_{4}$ v'è uno spigolo di estremi $b$ e $y$ (ovviamente, se in $G$ per un certo $y$ vi sono due vertici $x_{i}$ adiacenti, lo spigolo di $G_{4}$ di estremi $b$ e $y$ è unico). Il grafo $G_{4}$ risolve il problema:

a) l'applicazione $g$ di $V(G)$ in $V\left(G_{4}\right)$ tale che $g(x)=x$ se, in $K, x$ non ha colore $A$, mentre $g(x)=b$ se il colore di $x$ è $A$ definisce un omomorfismo di $G$ in $G_{4}$ : esso è suriettivo, poichè ogni vertice di $G_{4}$ è immagine d'un vertice di $G$, mentre uno spigolo di $G_{4}$ è immagine di se stesso, oppure - se uno dei suoi estremi è $b-$ è immagine di se stesso o d'uno spigolo un cui estremo ha colore $A$.

b) $G_{4}$ ha numero cromatico $k-1$. Infatti, $K$ dà una sua colorazione con $k-1$ colori. D'altra parte, non esiste una colorazione di $G_{4}$ con meno di $k-1$ colori: se $K^{\prime}$ fosse una tale colorazione, dando a ogni vertice di $G$ che appartiene anche a $G_{4}$ il suo colore in $K^{\prime}$ e ai vertici $x_{i}$ (che a due a due non sono adiacenti) un nuovo colore, si avrebbe una colorazione di $G$ con meno di $k$ colori, contro l'ipotesi.

5) Trovare un grafo $G_{5}$ di numero cromatico $h>k$ e un omomorfismo iniettivo di $G$ in $G_{5}$.

Il grafo $G_{3}$, e l'immersione di $G$ in $G_{3}$, risolvono manifestamente il problema.

Notiamo infine che non esiste un grafo di numero cromatico maggiore di $k$ di cui $G$ sia immagine omomorfa iniettiva, nè uno di numero eromatico inferiore a $k$ che sia immagine iniettiva omomorfa di $G$. Infatti, se $f$ è un omomorfismo iniettivo di $G^{\prime}$ in $G^{\prime \prime}, f\left(G^{\prime}\right)$ è un sottografo di $G^{\prime \prime}$, isomorfo a $G^{\prime}$ : il suo numero cromatico, che è quello di $G^{\prime}$, non può superare quello di $G^{\prime \prime}$.

Inoltre, non esiste una regola che associ a ogni grafo uno di numero cromatico superiore, che ne sia immagine omomorfa suriettiva. Infatti, se esistesse una tale 
regola, applicandola successivamente a un grafo finito, il numero cromatico aumenterebbe, mentre il numero dei vertici non aumenterebbe. Percio, dopo un numero finito di passaggi, si otterrebbe un grafo il eui numero cromatico è superiore al numero dei vertici, il che è assurdo.

In casi particolari, tuttavia, è possibile, dato un grafo, trovarne un'immagine suriettiva omomorfa di numero cromatico superiore. Ad esempio, dato il grafo $G$ tale che $V(G)=\{a, b, c, d\}, \mathbb{S}(G)=\{q, r, s\}$, con $\varphi(q)=\{a, b\}, \varphi(r)=\{b, c\}, \varphi(s)=$ $=\{c, d\}$, che ha numero cromatico 2 , e il grafo completo $G^{\prime}$ (senza lacei) costruito sui vertici $a^{\prime}, b^{\prime}, e^{\prime}$, di numero cromatico 3 , l'applicazione di $V(G)$ su $V\left(G^{\prime}\right)$ tale che $g(a)=g(d)=a^{\prime}, g(b)=b, g(c)=c^{\prime}$ definisce un omomorfismo suriettivo di $G$ su $G^{\prime}$.

Si osservi che gli omomorfismi che risolvono i vari problemi sono tutti connessi (cioè le antiimmagini d'un vertice sono grafi connessi), salvo al più quello del problema 4). Gli omomorfismi suriettivi sono forti (cioè, ogni coppia di vertici adiacenti nel codominio è immagine d'almeno una coppia di vertici adiacenti). Si osservi che con le costruzioni precedenti si sono risolti i problemi seguenti: dato un grafo $G$ di numero cromatico finito e un numero $h \in N_{0}$, trovare un grafo di numero cromatico $h$ che ne sia immagine omomorfa, e uno di cui $G$ sia immagine omomorfa.

3. - Finora ci siamo riferiti a proprietà relative al numero cromatico d'un grafo: ora ci occuperemo di grafi colorati, cioè di terne $(G, H, K)$, dove $G$ è un grafo non orientato, $H$ è un insieme (i cui elementi si diranno colori) e $K$ è un'applicazione di $\nabla(G)$ in $H$ (detta colorazione: $K$ potrebbe non essere suriettiva, contrariamente a quanto si suppone solitamente) tale che l'antiimmagine di ogni $A \in H$ sia un insieme stabile di vertici. In questo numero, $H$ può essere finito o infinito.

Siano $O=(G, H, K)$ e $O^{\prime}=\left(G^{\prime}, H^{\prime}, K^{\prime}\right)$ grafi colorati e sia $h: H \rightarrow H^{\prime}$ un'applicazione. Un omomorfismo semi-colorato relativo $a$ h è un omomorfismo $f$ di $G$ in $G^{\prime}$ tale che il diagramma seguente commuti:

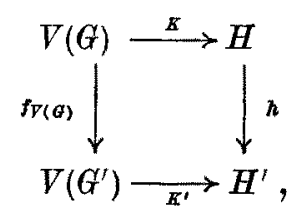

vale a dire, $K^{\prime} \circ f_{V(G)}=h \circ K\left[f_{V(G)}: V(G) \rightarrow V\left(G^{\prime}\right)\right.$ è la restrizione di $f$ a $\left.V(G)\right]$. Se $H=H^{\prime}$ e $h$ è l'identità $1_{H}, f$ si dirà un omomorfismo colorato (in esso, vertici associati hanno il medesimo colore).

Fissiamo un insieme $H$, e un monoide (con elemento neutro) $(\Omega, \circ)$ subordinato al monoide $(\mathscr{F}(H, H)$, o) delle applicazioni di $H$ in sè. Proviamo che:

Esiste una categoria $\mathbf{C o l}_{\Omega}^{H}$ che ha come oggetti $i$ grafi colorati con colori $d i H$ $H$ come morfismi le quaterne $\left(C, C^{\prime}, f, h\right)$, dove $f$ è un omomorfismo semi-colorato $d i \quad C$ in $C^{\prime \prime}$ relativo a $h$. Associando al morfismo $\left(C, C^{\prime}, f, h\right)$ l'applicazione $h$ si ha un funtore $F$ di $\operatorname{Col}_{(\mathbb{H}, \mathbb{B})}^{B}$ sul monoide $(\mathcal{F}(H, H), 0)$. 
Siano $C=(G, H, K), O^{\prime}=\left(G^{\prime}, H, K^{\prime}\right), O^{\prime \prime}=\left(G^{\prime \prime}, H, K^{\prime \prime}\right)$ grafi colorati, e sia $h, h^{\prime} \in \Omega$. Siano $f(h), f\left(h^{\prime}\right)$ omomorfismi semi-colorati di $C$ in $C^{\prime}$ e di $C^{\prime}$ in $C^{\prime \prime}$ rispettivamente, relativi a $h$ e a $h^{\prime}$ rispettivamente. Dalle uguaglianze

$$
\begin{aligned}
& K^{\prime} \circ f(h)_{\mathcal{V}\left(G^{\prime}\right)}=h \circ K, \\
& K^{\prime \prime} \circ f\left(h^{\prime}\right)_{V\left(G^{\prime}\right)}=h^{\prime} \circ K^{\prime},
\end{aligned}
$$

componendo la seconda a destra con $f(h)_{V(G)}$ si ottiene

$$
K^{\prime \prime} \circ f\left(h^{\prime}\right)_{V\left(G^{\prime}\right)} \circ f(h)_{V(G)}=h^{\prime} \circ K^{\prime} \circ f(h)_{V(G)}=h^{\prime} \circ h \circ K .
$$

Questa si può anche serivere

$$
K^{\prime \prime} \circ\left(f\left(h^{\prime}\right) \circ f(h)\right)_{V(G)}=\left(h^{\prime} \circ h\right) \circ K,
$$

che ci dice ehe il prodotto di $f(h)$ e di $f\left(h^{\prime}\right)$ è l'omomorfismo semi-colorato $f\left(h^{\prime} \circ h\right)$, dove $h^{\prime} \circ h \in \Omega$. Per ogni $C$, si ha poi che $\left(C, C, 1_{G}, 1_{H}\right)$ è un omomorfismo semi-colorato (anzi, colorato): dunque si ha una categoria, con la composizione

$$
\left(C^{\prime}, C^{\prime \prime}, f\left(h^{\prime}\right), h^{\prime}\right) *\left(C, C^{\prime}, f(h), h\right)=\left(O, O^{\prime \prime}, f\left(h^{\prime} \circ h\right), h^{\prime} \circ h\right) .
$$

Si è pure visto che $f\left(h^{\prime}\right) \circ f(h)$ è relativo a $h^{\prime} \circ h$, mentre l'elemento neutro $(C, C$, $\left.1_{G}, 1_{H}\right)$ è relativo all'elemento neutro $1_{H}$ di $\Omega$. Si ha dunque un funtore della categoria $\operatorname{Col}_{\mathfrak{F}^{\prime}(H, H)}^{\bar{H}}$ in $(\mathscr{F}(H, H), \circ)$. Tale funtore è suriettivo. Infatti, per ogni applicazione $h: H \rightarrow H$, consideriamo i grafi completi (senza lacci) $G, G^{\prime}$ tali che $V(G)=$ $=H, \nabla\left(G^{\prime}\right)=h(H)$, con le colorazioni $1_{H}$ e $j_{h(H)}$ (quest'ultima è l'iniezione canonica di $h(H)$ in $H)$ : l'applicazione $h$ definisce un omomorfismo semi-colorato del primo sul secondo, relativo a $h$.

In modo analogo, per ogni $\Omega \subseteq \mathscr{F}(H, H)$, si ha un funtore, subordinato a $F$, di $\operatorname{Col}_{\Omega}^{H}$ su $(\Omega, o)$.

Sia ancora $f$ un omomorfismo semi-colorato di $C=(G, H, K)$ in $C^{\prime}=\left(G^{\prime}, H^{\prime}, K^{\prime}\right)$, relativo a un'applicazione $h$. Se $f$ è biiettivo, allora anche $f^{-1}$ è un omomorfismo di $G^{\prime}$ su $G$ (ed $f$ è un isomorfismo di $G$ su $G^{\prime}$ ) [4]. Se anche $h$ è biiettiva, $f^{-1}$ è un omomorfismo semi-colorato di $O^{\prime}$ su $C$, relativo a $h^{-1}$, poichè da

segue in tal caso

$$
K^{\prime} \circ f_{\bar{V}(\theta)}=h \circ K
$$

$$
h^{-1} \circ K^{\prime}=K \circ f_{\boldsymbol{V}(G)}^{-1}
$$

In tal caso si dirà che $f$ è un isomorfismo semi-colorato di $C$ su $C^{\prime}$. Se $f$ è un omomorfismo colorato biiettivo di $C$ su $C^{\prime}$, anche $f^{-1}$ è un omomorfismo colorato, e $f$ si dirà un isomorfismo colorato.

Al solito, un automorfismo è un isomorfismo di una struttura su se stessa. 
Gli automorfismi semi-colorati di un grafo colorato $C$ formano un gruppo $\Delta(C)$, e gli automorfismi colorati formano un gruppo $\Phi(C)$ : sia poi $\Gamma(C)$ il gruppo degli automorfismi del grafo $G$ tale che $C=(G, H, K)$ (se non v'è pericolo di confusione, i tre gruppi si indicheranno rispettivamente con $\Delta, \Phi, \Gamma$ ). Ovviamente

$$
\Phi \subseteq \Delta \subseteq \Gamma .
$$

Sia poi $(S, \circ)$ un gruppo di biiezioni di $H$ in sè. Gli automorfismi semi-colorati relativi a una $h \in S$ formano un gruppo $\Delta_{S}(C)$ (o semplicemente $\Delta_{S}$ ). Manifestamente

$$
S^{\prime} \subseteq S \Rightarrow \Delta_{\mathcal{S}^{\prime}} \subseteq \Delta_{S} .
$$

Fissato un grafo colorato $C$, applichiamo il funtore $F$ ai morfismi invertibili di Hom $(C, C)$ : abbiamo un omomorfismo di $A(C)$ sul gruppo $\left(\mathscr{S}_{H}, \circ\right)$ delle biiezioni di $H$ in sè. Quindi, se $(S, \circ)$ è un sottogruppo invariante di $\left(\mathscr{S}_{H}, \circ\right), A_{S}(C)$ è sottogruppo invariante di $\Delta(C)$ : in particolare, $\Phi(C)$ è sottogruppo invariante $d i \Delta(C)$.

4. - In questo numero ei occuperemo prevalentemente della determinazione di grafi colorati i cui gruppi $\Gamma, \Delta, \Phi$ soddisfano particolari condizioni.

Si noti che, in un grafo completo $G$, in ogni colorazione due vertici debbono avere colori differenti, e quindi (se l'ordine è $>1$ )

$$
\Gamma(C)=\Delta(C) \neq \Phi(C)=\left\{1_{a}\right\} .
$$

La (1) è tuttavia valida anche per il grafo della figura 1 (qui e nelle figure successive le lettere maiuscole indicano i colori attribuiti ai vertici, mentre le eventuali lettere minuscole sono i nomi dei vertici).

Nel grafo colorato della figura 2 si ha $\Gamma \neq \Delta=\Phi$, mentre in quello della figura 3 abbiamo $\Gamma \neq \Delta \neq \Phi$ : infatti, in quest'ultimo, la biiezione

$$
\left(\begin{array}{llllll}
a & b & c & h & k & l \\
h & k & l & a & b & o
\end{array}\right)
$$

definisce un omomorfismo che non appartiene a $\Delta$, mentre la biiezione

$$
\left(\begin{array}{llllll}
a & b & c & h & k & l \\
b & c & a & k & l & h
\end{array}\right)
$$

definisce un omomorfismo che appartiene a $\Delta$ ma non a $\Phi$.

Per un "ciclo" o per una "catena" d'ordine $2 n$, colorati con il minimo numero di colori (cioè con 2 colori), si ha $\Gamma=\Delta \neq \Phi$ (nel primo caso, $\Gamma=\Delta$ e $\Phi$ sono iso-

24 - Annali di Matematica 


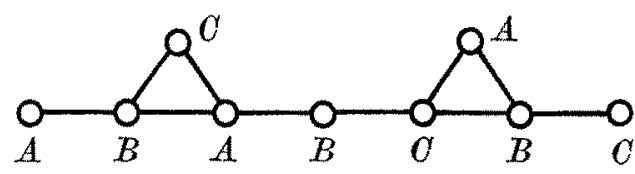

Figura 1

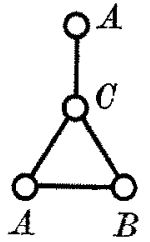

Figura 2

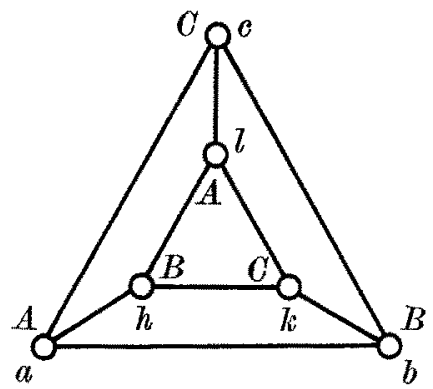

Figura 3

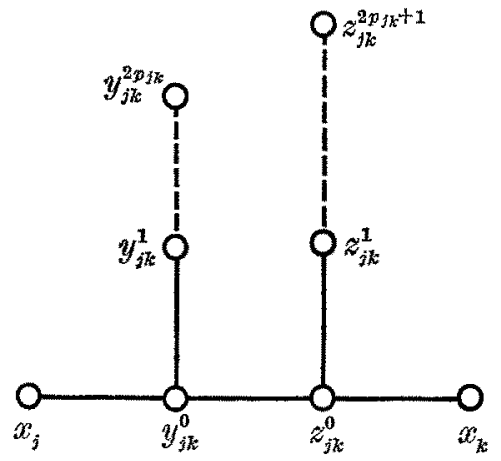

Figura 4

morfi rispettivamente a $\boldsymbol{D}_{2 n}$ e a $\boldsymbol{D}_{n}$, nel secondo $\Gamma$ e $\Delta$ sono isomorfi $a\left(\boldsymbol{Z}_{2},+\right)$ mentre $\Phi$ s'annulla). Nel caso d'una catena d'ordine dispari $\Gamma=\Delta=\Phi$ (sono tutti isomorfi a $\left.\left(Z_{2},+\right)\right)$.

A questo proposito, possiamo esprimere una condizione caratteristica affinchè un dato grafo $G$ si possa colorare in modo tale che i suoi gruppi $\Gamma$ e $\Phi$ (e quindi anche $\Delta)$ coincidano. Diciamo insiemi di transitività i sottoinsiemi di $V(G)$ che danno luogo alla partizione associata alla relazione d'equivalenza

"esiste una $f \in \Gamma$ tale che $y=f(x) »$.

Ciascuno di essi descrive, con gli spigoli che in $Q$ conginngono i suoi vertici, un sottografo di $G$. Ebbene:

Condizione necessaria e sufficiente affinchè un grafo $G$ si possa colorare in modo che $\Gamma=\Delta=\Phi$ è che $i$ suoi insiemi di transitività siano stabili.

Infatti, se esiste una colorazione di $G$ tale che $\Gamma=\Phi$, ogni $f \in \Gamma$ è un omomorfismo colorato, perciò due vertici $a, b$ tali che $b=f(a)$ hanno il medesimo colore e quindi non sono adiacenti. Viceversa, sia $G$ un grafo tale che i vertici di uno stesso insieme di transitività siano a due a due non adiacenti: essi si possono colorare con il medesimo colore, perciò ogni automorfismo è un automorfismo colorato per una colorazione siffatta: dunque $\Gamma=\Phi$ e anche $\Delta=\Gamma$. 
Si osservi che, dato un grafo, si può sempre colorarlo in modo che $\Gamma=\Delta$ : basta assegnare colori distinti a vertici distinti. Invece, vi sono dei grafi che non si possono colorare in modo che $A=\Phi$ (si pensi ad esempio a un grafo completo di ordine $>2$ ).

Si possono porre i problemi seguenti: dato un gruppo $\gamma$, determinare un grafo colorato $C$ tale che $\Delta(O)=\gamma$, oppure tale che $\Phi(C)=\gamma$. La costruzione seguente risolve entrambi i problemi nel caso in cui $\gamma$ sia finito o numerabile.

Ricordiamo la costruzione di FRUCHT ([2], [3]) d'un grafo non orientato $F(\gamma)$ il cui gruppo degli automorflsmi è isomorfo al gruppo dato $\gamma$ (di cardinalità $\leqslant \aleph_{0}$ ). Numeriamo gli elementi di $\gamma, x_{1}, x_{2}, \ldots$, e, per ogni eoppia $\left(x_{j}, x_{k}\right)$, sia $p_{j k}$ il numero tale che $x_{p_{j k}}=x_{j}^{-1} x_{k}$. Consideriamo il grafo orientato completo (ma senza lacei) di vertici $x_{1}, x_{2}, \ldots$, e sostituiamo allo spigolo di primo estremo $x_{j}$ e secondo estremo $x_{k}$ il grafo non orientato rappresentato nella figura 4: si ottiene cosi un grafo non orientato $F$, il cui gruppo degli automorfismi è isomorfo a $\gamma$.

Osserviamo che il grafo $F$ soddisfa la condizione necessaria e sufficiente affinchè lo si possa colorare in modo che $\Gamma=\Phi$. Infatti, in un automorfismo di $F$ un vertice del tipo $x_{j}$ si trasforma in uno dello stesso tipo, e così pure per quelli del tipo $y_{1 k}^{\alpha}$ e anche per quelli del tipo $z_{j k}^{\alpha}$ (che si distinguono dai precedenti in quanto appartengono a una "catena" di lunghezza dispari). Inoltre, due vertici $x_{j}$ non sono mai adiacenti, e così pure due vertici $y_{j k}^{\alpha}$ trasformati l'uno nell'altro da un automorfismo di $F$, in quanto corrispondono a valori differenti di $j$ o di $k$ (altrettanto dicasi per i vertici $z_{j k}^{\alpha}$ ). Ciascun insieme di transitività è dunque stabile.

Se $\gamma$ ha più di due elementi, il grafo $F$ non è bicromatico. In ogni caso possiamo però dare la seguente colorazione (con 3 colori), per la quale $\Gamma=\Phi$. Ai vertici $x_{j}$ attribuiamo il colore $A$; ai vertici $y_{i k}^{2 \beta}$ e $z_{i k}^{2 \beta+1}$ il colore $B$; ai vertici $y_{j k}^{2 \beta+1}$ e $z_{j k}^{2 \beta}$ il colore $C$. Ogni automorfismo del grafo $F$ è un automorfismo del grafo colorato cosi ottenuto, e il gruppo degli automorfismi semi-colorati e quello degli automorfismi colorati coincidono, e sono isomorfi a $\gamma$.

\section{BIBLIOGRAFIA}

[1] C. BERGe, Graphes et hypergraphes, Dunod, Paris, 1970.

[2] H. FRUCHT, Herstellung von Graphen mit vorgegebener abstrakter Gruppe, Compositio Math., 6 (1938), pp. 239-250.

[3] O. Ore, Theory of graphs, Amer. Math. Soc. Coll. Publ., Providenee, 1962.

[4] F. SPenanzA, Omomorfismi fra grafi e grafi moltiplicativi, Ann. di Mat., (4), 71 (1966), pp. 281-294.

[5] F. SPERANZA, Sur les sections des hypergraphes et sur leurs automorphismes, Coll. Math., 27 (1973), pp. 269.274 . 